\title{
Nuclear expression of p53 in mature tumor endothelium of retinoblastoma
}

\author{
BYUNG JOO LEE ${ }^{1,2}$, JIN HYOUNG KIM ${ }^{1,3}$, DONG HYUN JO ${ }^{1-3}$, KYU-WON KIM ${ }^{3,4}$, \\ YOUNG SUK YU ${ }^{1,2}$ and JEONG HUN KIM ${ }^{1-3}$
}

\author{
${ }^{1}$ Fight Against Angiogenesis-Related Blindness (FARB) Laboratory, Clinical Research Institute, \\ Seoul National University Hospital, Seoul; ${ }^{2}$ Department of Ophthalmology, College of Medicine, \\ Seoul National University, Seoul; ${ }^{3}$ Tumor Microenvironment Research Center, Global Core Research Center, \\ Seoul National University, Seoul; ${ }^{4}$ Neurovascular Coordination Research Center, College of Pharmacy and \\ Research Institute of Pharmaceutical Sciences, Seoul National University, Seoul, Republic of Korea
}

Received April 7, 2014; Accepted May 16, 2014

DOI: 10.3892/or.2014.3236

\begin{abstract}
The present study aimed to investigate the p53 expression pattern in tumor cells and in mature tumor vascular endothelium of retinoblastoma. Nuclear p53 accumulation was observed in most of the tumor cells in both the human and orthotopic retinoblastoma animal models using SNUOT-Rb1 and Y79 cells. In the orthotopic animal model, some of the tumor vascular endothelium also demonstrated nuclear p53 immunoreactivity, and the ratio of 553 positivity among the total mature tumor vascular endothelium was slightly higher in the Y79 cell model when compared with the SNUOT-Rb1 cell model. In addition, in the human retinoblastoma specimens, $32.9 \%$ of the tumor vascular endothelium showed p53 nuclear staining. In conclusion, some of the mature tumor vascular endothelium in both the human and orthotopic models of retinoblastoma share the same cytogenetic abnormality (an abnormal nuclear accumulation of p53) with retinoblastoma cells.
\end{abstract}

\section{Introduction}

Retinoblastoma is a hypervascular tumor, the proliferation of which is dependent on the vascular supply of the tumor (1). It is also known that the vascular density of human retinoblastoma

Correspondence to: Professor Jeong Hun Kim, Fight Against Angiogenesis-Related Blindness Laboratory, Biomedical Research Institute, Seoul National University Hospital, 101 Daehak-Ro, Jongno-Gu, Seoul 110-744, Republic of Korea

E-mail: steph25@snu.ac.kr

Professor Young Suk Yu, Department of Ophthalmology, College of Medicine, Seoul National University, 103 Daehak-ro, Jongno-gu, Seoul 110-799, Republic of Korea

E-mail:ysyu@snu.ac.kr

Key words: endothelial cells, nuclear expression, p53, retinoblastoma, tumor vessels is closely related to the local invasion and distant metastasis of the disease (2). Although local therapy and systemic chemotherapy are widely adopted for ocular salvage, enucleation is still regarded as the treatment of choice in advanced cases of retinoblastoma. Since the inhibitory effects of several anti-angiogenic agents such as anecortave acetate (3), bevacizumab (4) and PEDF (5) on tumor growth have been proven in transgenic and orthotopic murine models of retinoblastoma, vascular targeted therapy is considered as one of the future adjuvant treatment options for retinoblastoma.

Since differences in gene expression between tumor vascular endothelium and normal endothelial cells have been noted, the concept of tumor vascularization by sprouting has changed. In human glioblastoma and neuroblastoma, chromosomal analysis using fluorescence in situ hybridization (FISH) revealed that some tumor vascular endothelium shows cytogenetic abnormality similar to that of tumor cells $(6,7)$. Moreover, direct evidence of tumor vascularization by the endothelial differentiation of cancer stem-like cells was found in glioblastoma $(7,8)$.

The $\mathrm{p} 53$ protein is well known as a tumor suppressor, which inhibits tumorigenesis through the activation of apoptosis and induction of cell cycle arrest in various types of human cancers including retinoblastoma (9). When stained with the DO-1 monoclonal antibody, $91.4 \%$ of specimens were found to exhibit positive staining, and the immunoreactivity to the p53 antibody was prominent in poorly or moderately differentiated areas (9). According to another study which explored the distribution of wild-type p53 in cultivated retinoblastoma cell lines and human retinoblastoma tissue using the p1801 monoclonal antibody (10), nuclear staining of the p53 protein was noted in $89 \%$ of human retinoblastoma. In contrast, retinoblastoma cell lines showed cytoplasmic or mixed nuclear and cytoplasmic distribution of the p53 protein (10).

Since tumor vascularization is also an important issue in the prognosis and treatment of retinoblastoma and there is increasing evidence of tumor stem-like cells in human and mouse retinoblastoma $(11,12)$, it is necessary to identify the presence of genetically unstable tumor vascular endothelium in retinoblastoma. In the present study, to investigate cytogenetic 
abnormalities in tumor endothelium of retinoblastoma, we examined the expression and distribution of the p53 protein in mature tumor vascular endothelium in an orthotopic retinoblastoma model and in human retinoblastoma.

\section{Materials and methods}

Retinoblastoma cells. Two types of human retinoblastoma cell lines were used in this study. SNUOT-Rb1 (13), which was established by our group, and Y79 (14) (American Type Culture Collection, Manassas, VA, USA) were cultured in RPMI-1640 media (Welgene Inc., Korea) supplemented with $10 \%$ fetal bovine serum (Gibco Invitrogen Corp., Carlsbad, CA, USA) and $1 \%$ penicillin-streptomycin solution (Invitrogen, Carlsbad, CA, USA). SNUOT-Rb1 cells maintained by our group were used at passage 3-7.

Orthotopic transplantation model. For induction of the orthotopic retinoblastoma mouse model, cultivated SNUOT-Rb1 and Y79 cells were injected into the vitreal cavity of BALB/cnude mice (Samtako, Osan, Korea) as previously described by our group (13). Three mice were assigned to each treatment group. The right eyes of the mice were injected with cultivated retinoblastoma cells $\left(1 \times 10^{7}\right)$ suspended in phosphate-buffered saline $\left(4^{\circ} \mathrm{C}\right)$, using a 30 -gauge needle. For the control, phosphate-buffered saline $\left(4^{\circ} \mathrm{C}\right)$ was injected into the left eyes of the mice in the same manner. Intraocular tumorigenesis was evaluated every week by indirect ophthalmoscope, and mice with adequate tumorigenesis were sacrificed and enucleated at the 4th week after injection. Mice were maintained and treated in accordance with the ARVO Statement for the Use of Animals in Ophthalmic and Vision Research.

Human retinoblastoma tissue. Formalin-fixed, paraffinembedded human eyeball sections $(4 \mu \mathrm{m})$ of three patients primarily enucleated for retinoblastoma were obtained from the Department of Pathology, Seoul National University Hospital. The Institutional Review Board of Seoul National University Hospital approved the study.

Double immunofluorescence staining. Formalin-fixed, paraffin-embedded blocks of tumor sections $(4-\mu \mathrm{m})$ (from both the orthotopic model and human retinoblastoma specimens) were deparaffinized by serial xylene and ethyl alcohol immersion. For antigen retrieval, sections were treated with proteinase $\mathrm{K}(20 \mu \mathrm{g} / \mathrm{ml})$ at $37^{\circ} \mathrm{C}$ for $20 \mathrm{~min}$. After being permeabilized with Triton X-100 (0.2\%), sections were blocked with blocking solution (BioGenex Laboratories, San Ramon, CA, USA). Prepared sections were incubated overnight with primary antibodies at $4^{\circ} \mathrm{C}$ and then with fluorescein tagged secondary antibody for $2 \mathrm{~h}$ at room temperature after rinsing. The anti-p53 polyclonal antibody (FL-393; sc-6243-G) and anti-von Willebrand factor (vWF) antibody (sc-14014) (both from Santa Cruz Biotechnology, Santa Cruz, CA, USA), for detecting mature vascular endothelium, were used as primary antibodies at the concentration of 1:100. For the secondary antibody, Alexa Fluor 594-conjugated donkey anti-goat IgG and Alexa Fluor 488-conjugated donkey anti-rabbit IgG (both from Molecular Probes, Carlsbad, CA, USA) were used at the concentration of 1:200. Nuclear counterstaining with 4,6-diamidino-2-phenylindole dihydrochloride (DAPI) was performed for the discrimination of nuclear or cytoplasmic distribution of the p53 protein. After aqueous mounting, the slides were examined under a fluorescence microscope (Axio Observer; Carl Zeiss). After confirming the number of total vWF-positive endothelium, the vWF-positive endothelium with p53 nuclear accumulation was independently evaluated by two investigators in 10 randomly selected high power fields (x400). Then, the overall ratio of endothelium with p53 nuclear accumulation among all vWF-positive tumor vascular endothelium was calculated.

Statistical analysis. Statistical analysis was performed using SPSS version 12.0 for Windows. The ratio of vWF-positive endothelium with p53 nuclear accumulation among all tumor vascular endothelial cells in the SNUOT-Rb1 and Y79 cell groups was compared using the Mann-Whitney U test. The cut-off value of statistical significance was $\mathrm{p}<0.05$.

\section{Results}

Expression and distribution of the 553 protein in the orthotopic retinoblastoma model. Four weeks after intravitreal inoculation of retinoblastoma cells, we assessed intraocular tumor formation with indirect ophthalmoscopic examination and H\&E staining. Adequate tumorigenesis was achieved in both Y79 and SNUOT-Rb1 cell induced mouse models. Although not quantitatively analyzed, the orthotopic retinoblastoma model induced by SNUOT-Rb1 cells showed a more aggressive growth pattern such as relatively rapid growth and occasional extraocular extension compared to the Y79 cell induced model. We did not note any other significant differences in phenotype between the tumors induced by Y79 and SNUOT-Rb1 cells. In both of the orthotopic retinoblastoma models induced by Y79 and SNUOT-Rb1 cells, strong and uniform immunopositivity against the anti-p53 antibody was observed in the area filled with retinoblastoma cells (Fig. 1). In contrast, the retinas of the contralateral eyes did not exhibit significant immunoreactivity to the anti-p53 antibody.

Most of the tumor cells demonstrated p53 immunoreactivity and when merged with DAPI nuclear counterstaining, we noted the nuclear location of the p53 protein in both Y79 and SNUOT-Rb1 cell models.

Mature tumor vascular endothelium with 553 protein immunoreactivity in the orthotopic retinoblastoma model. Some of the vWF-immunopositive tumor vascular endothelium was also stained with anti-p53 antisera. In both Y79 and SNUOT-Rb1 models, the nuclear localization of the $\mathrm{p} 53$ protein in the vWFimmunopositive tumor vascular endothelium was confirmed by DAPI nuclear counterstaining. The distribution of the p53 protein in endothelial cells resembled that in the tumor cells (Fig. 2). In contrast to the tumor vascular endothelium in the retinoblastoma model, retinal vascular endothelium of the contralateral normal eyes did not react with p53 antisera.

The ratio of p53 immunopositivity was different in each orthotopic model induced by Y79 and SNUOT-Rb1 cells. The mean percentage of p53-positive endothelium was $31.4 \pm 5.4 \%$ in 3 eyes from the orthotopic transplantation model with SNUOT-Rb1 cells, and was $46.1 \pm 1.6 \%$ in the other 3 eyes from 

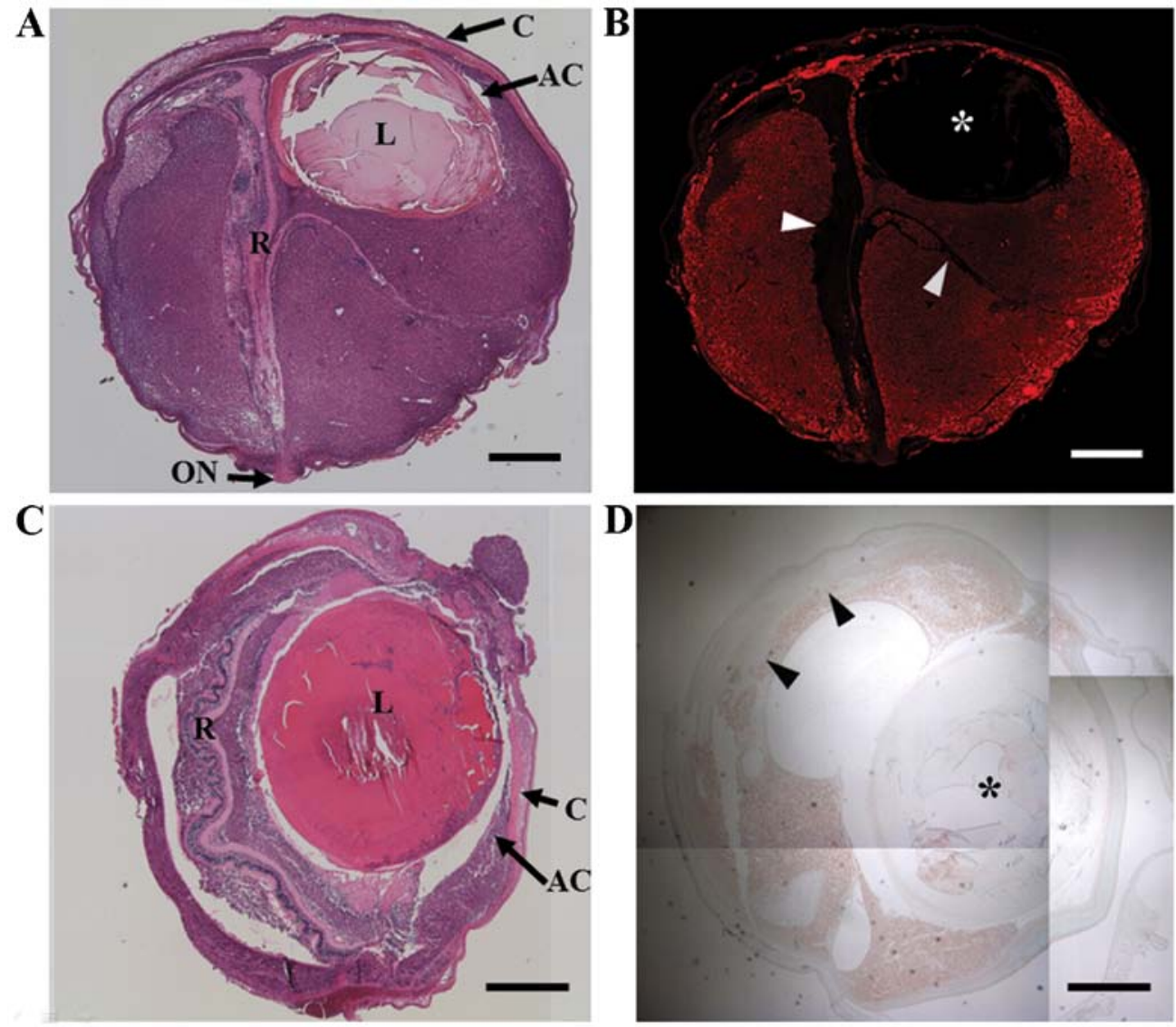

Figure 1. Diffuse p53 expression in an orthotopic transplantation mouse model of retinoblastoma. (A) A tumor mass filling the subretinal area, intravitreal cavity and anterior chamber can be observed 4 weeks after the intravitreal inoculation of SNUOT-Rb1 cells (H\&E staining). (B) In a serial section stained with fluorescein tagged anti-p53 antibody (red), diffuse p53 immunoreactivity of the tumor mass is noted apart from the detached retina (arrowheads) and crystalline lens $\left(^{*}\right)$. (C) After inoculation of Y79 cells, intraocular tumor formation similar to the SNUOT-Rb1 cell model was observed, but the tumor volume was relatively smaller in the Y79 model. (D) Immunohistochemical staining of the Y79 cell induced model also shows diffuse p53 immunopositivity of the tumor mass apart from the relatively preserved retina (arrow heads) and crystalline lens $\left(^{*}\right)$. AC, anterior chamber; C, cornea; L, lens; ON, optic nerve; R, detached retina. Scale bars, A-D, $500 \mu \mathrm{m}$.
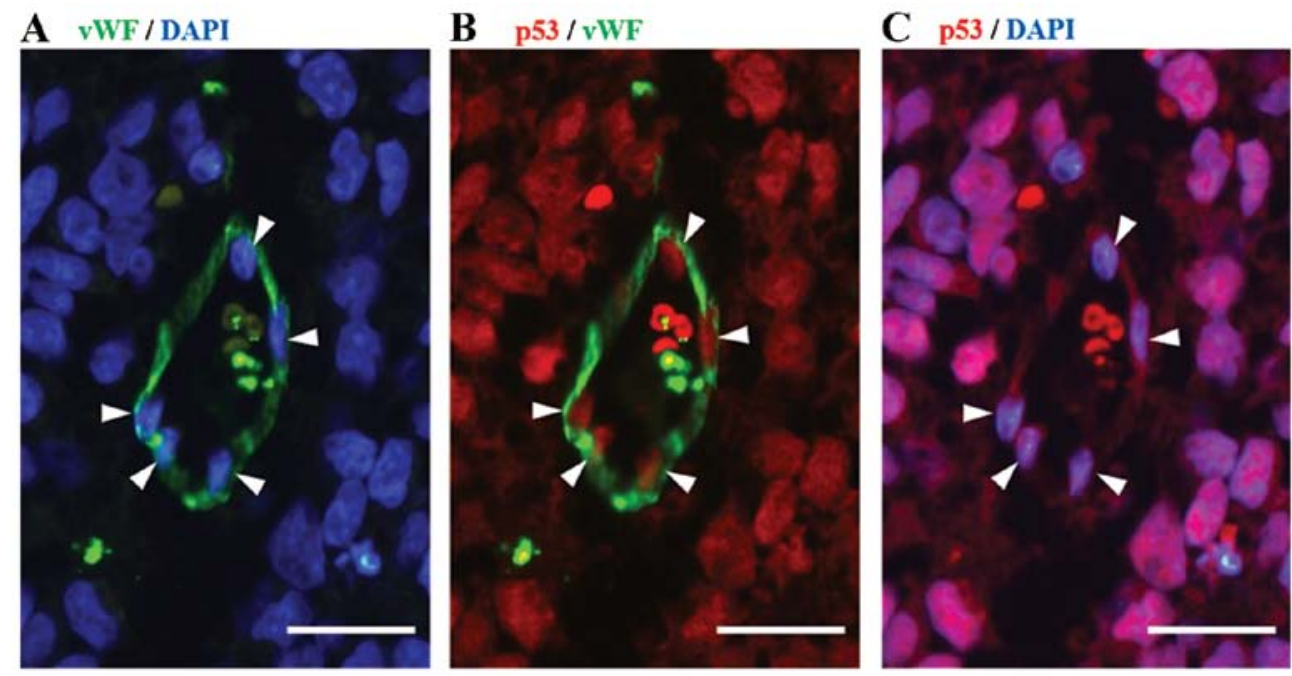

Figure 2. Double immunofluorescence staining with anti-p53 (red) and anti-vWF (green) antibodies demonstrates p53 nuclear accumulation in the mature tumor vascular endothelium of the orthotopic retinoblastoma model. After identifying the nuclei of mature tumor vascular endothelium (arrowheads) by DAPI (blue) counterstaining (A), nuclear accumulation of p53 was detected in these cells (B and C). Scale bars, $10 \mu \mathrm{m}$. vWF, von Willebrand factor.

the Y79 cell model (Fig. 3). The ratio of tumor vascular endothelium with p53 nuclear accumulation was higher in the Y79 cell induced orthotopic retinoblastoma model with a marginal statistical significance $(\mathrm{p}=0.05)$. 
Table I. Clinicopathological characteristics of the human retinoblastoma cases.

\begin{tabular}{|c|c|c|c|c|c|c|c|c|c|c|}
\hline \multirow[b]{2}{*}{$\begin{array}{l}\text { Patient } \\
\text { no. }\end{array}$} & \multirow[b]{2}{*}{$\begin{array}{c}\text { Age } \\
\text { (months)/ } \\
\text { gender }\end{array}$} & \multirow[b]{2}{*}{ Laterality } & \multirow[b]{2}{*}{$\begin{array}{l}\text { International } \\
\text { classification }\end{array}$} & \multirow[b]{2}{*}{$\begin{array}{l}\text { Growth } \\
\text { pattern }\end{array}$} & \multirow[b]{2}{*}{$\begin{array}{l}\text { MTD } \\
(\mathrm{mm})\end{array}$} & \multicolumn{3}{|c|}{ Gross pathology } & \multicolumn{2}{|c|}{$\begin{array}{l}\text { Percentage of p53- } \\
\text { positive endothelium }\end{array}$} \\
\hline & & & & & & $\begin{array}{c}\text { AC } \\
\text { involve- } \\
\text { ment }\end{array}$ & $\begin{array}{c}\text { Choroidal } \\
\text { involve- } \\
\text { ment }\end{array}$ & $\begin{array}{c}\text { Optic nerve } \\
\text { involve- } \\
\text { ment }\end{array}$ & $\begin{array}{l}\text { Observer } 1 \\
(\%)\end{array}$ & $\begin{array}{c}\text { Observer } 2 \\
(\%)\end{array}$ \\
\hline 1 & $8 / \mathrm{M}$ & Bilateral & Group D & Exophytic & 15 & No & No & No & 29.3 & 33.3 \\
\hline 2 & $12 / \mathrm{M}$ & Unilateral & Group D & Exophytic & 16 & No & Yes & No & 31.0 & 36.2 \\
\hline 3 & 9/M & Unilateral & Group D & Exophytic & 13 & No & No & No & 32.4 & 35.1 \\
\hline
\end{tabular}

$\mathrm{AC}$, anterior chamber; MTD, maximal tumor diameter.

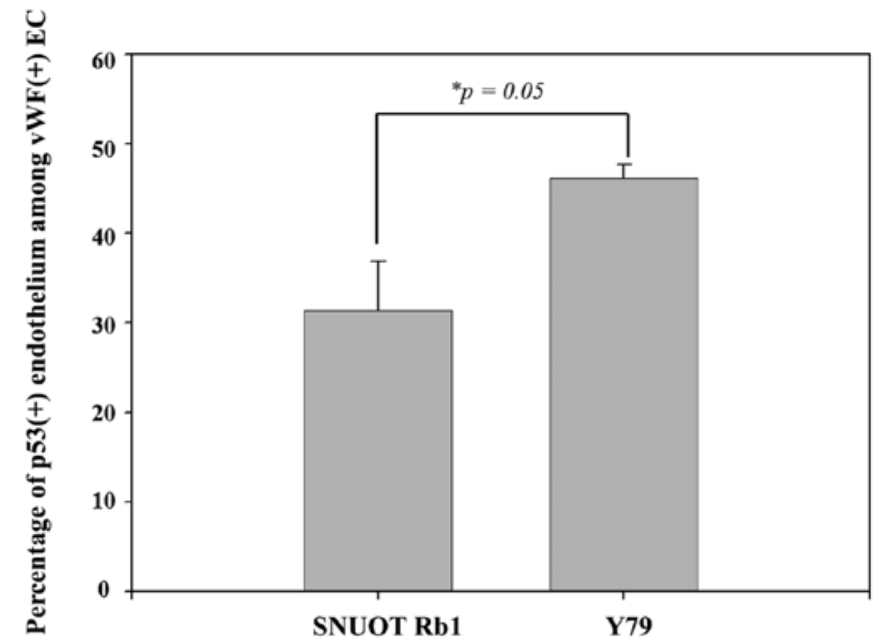

Figure 3. Comparison of the percentage of mature tumor vascular endothelium which exhibited p53 nuclear accumulation in the orthotopic retinoblastoma models induced by SNUOT-Rb1 and Y79 cells. The percentage of p53immunopositive endothelium was higher in the Y79 model with borderline statistical significance ("Mann-Whitney U test). EC, endothelial cells; vWF, von Willebrand factor.

Immunoreactivity of the human retinoblastoma specimens against the anti-p53 antibody: tumor cells and vascular endothelium. All three patients involved in this study were primarily enucleated for extensive retinoblastoma which was classified as group D according to the International Intraocular Retinoblastoma Classification. Extraocular extension was not detected in any patients at the time point of enucleation. The clinicopathological features of the patients were similar (Table I).

In the present study, human retinoblastoma cells demonstrated a variable immunoreactivity against the anti-p53 antibody (FL-393), showing a similar trend with the results of a previous study using the DO-1 monoclonal antibody (9). When comparing the distribution of p53 immunopositivity with DAPI nuclear counterstaining, the p53 protein in retinoblastoma cells was mainly nuclear, with some cytoplasmic localization. In cases of cytoplasmic p53 distribution, the immunoreactivity was typically weak. In every specimen, a fraction of vWF-positive tumor vascular endothelium presented nuclear accumulation of the p53 protein (Fig. 4).
In a specimen which had a portion of relatively preserved retinal layers, we found retinal vessels, presumed to be preexisting and not of neoplastic origin. To verify whether the endothelial p53 immunopositivity exclusively occurs in tumor vessels, we carefully reviewed the retinal vessels in the preserved retina. The vWF-positive endothelial cells of those vessels did not exhibit any p53 immunopositivity without exception (Fig. 5). The percentage of p53-positive endothelium among the total vWF-positive tumor vascular endothelium counted by two independent observers is presented in Table I. The average of the percentages reported by each observer ranged from 31.3 to $33.8 \%$.

\section{Discussion}

In human retinoblastoma, no genetic mutation of p53 was previously noted in the primary tumors (15). Yet, following a report by Laurie et al (16), it is generally accepted that in some human retinoblastoma cases, the p53 protein remains wild-type but is functionally inactivated by abnormally amplified $M D M 2$ and $M D M X$. According to their results, the amplification of $M D M X$ which does not have ubiquitin ligase activity is found in $65 \%$ of human retinoblastoma cases (17), whereas that of $M D M 2$ which results in ubiquitin-dependent p53 degradation is noted in only $10 \%$ of human retinoblastomas $(16,18)$.

Since several previous reports indicate that $\mathrm{p} 53$ nuclear staining of tumor cells is observed in most cases of human retinoblastoma $(9,10)$, the authors considered p53 nuclear staining as a putative marker of cytogenetic abnormality in the subjects with retinoblastoma $(9,10)$. A previous study concerning the p53 expression of normal mouse retina revealed that the entire layer of retina did not show any significant immunoreactivity against all three types of anti-p53 antibodies used for the immunofluorescence assay (including FL-393, which was used in this study) (19). In another study that evaluated the p53 expression of the normal mouse eye using anti-p53 driven CAT staining, only the photoreceptor layer showed significant p53 expression throughout the entire retinal layers (20). Since we also evaluated the contralateral eyes of the orthotopic model to rule out the possibility of detecting physiological p53, nuclear staining of p53 could be considered as a putative marker of cytogenetic abnormality. 
A 553

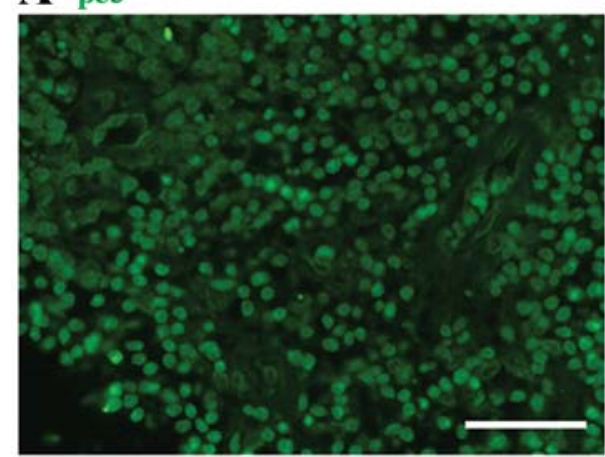

C p53/vWF

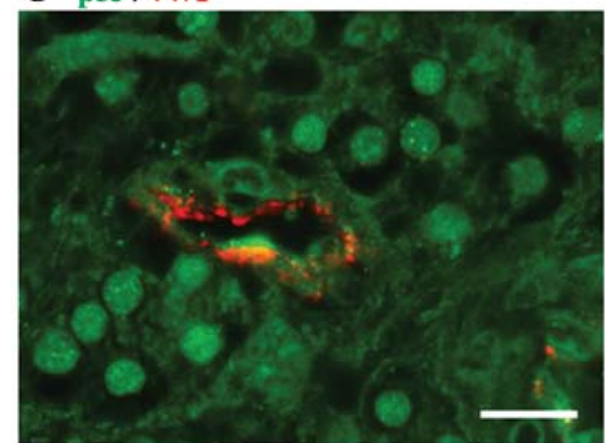

B p53/DAPI

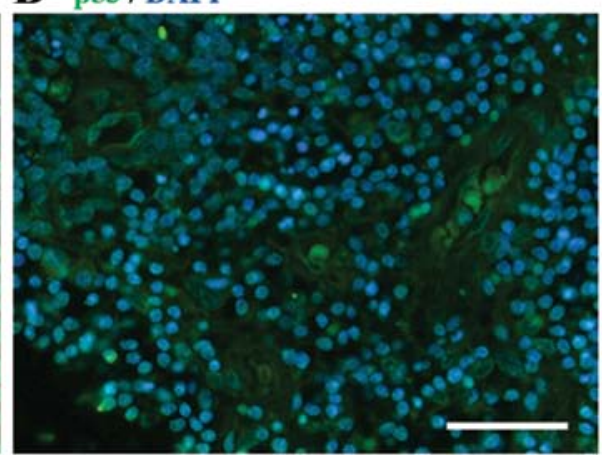

D $533 / \mathrm{vWF}$

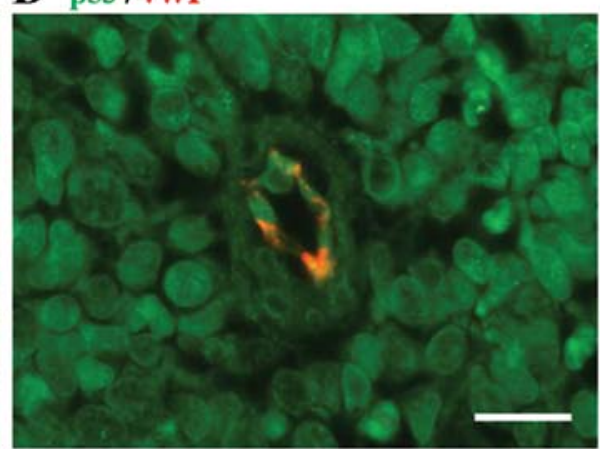

Figure 4. Double immunofluorescence staining with anti-p53 (green) and anti-vWF (red) antibodies demonstrates p53 nuclear accumulation in both tumor cells (A and B) and the mature tumor vascular endothelium (C and D) of human retinoblastoma tissue. Scale bars, A and B, $50 \mu \mathrm{m} ; \mathrm{C}$ and $\mathrm{D}, 10 \mu \mathrm{m}$. vWF, von Willebrand factor.

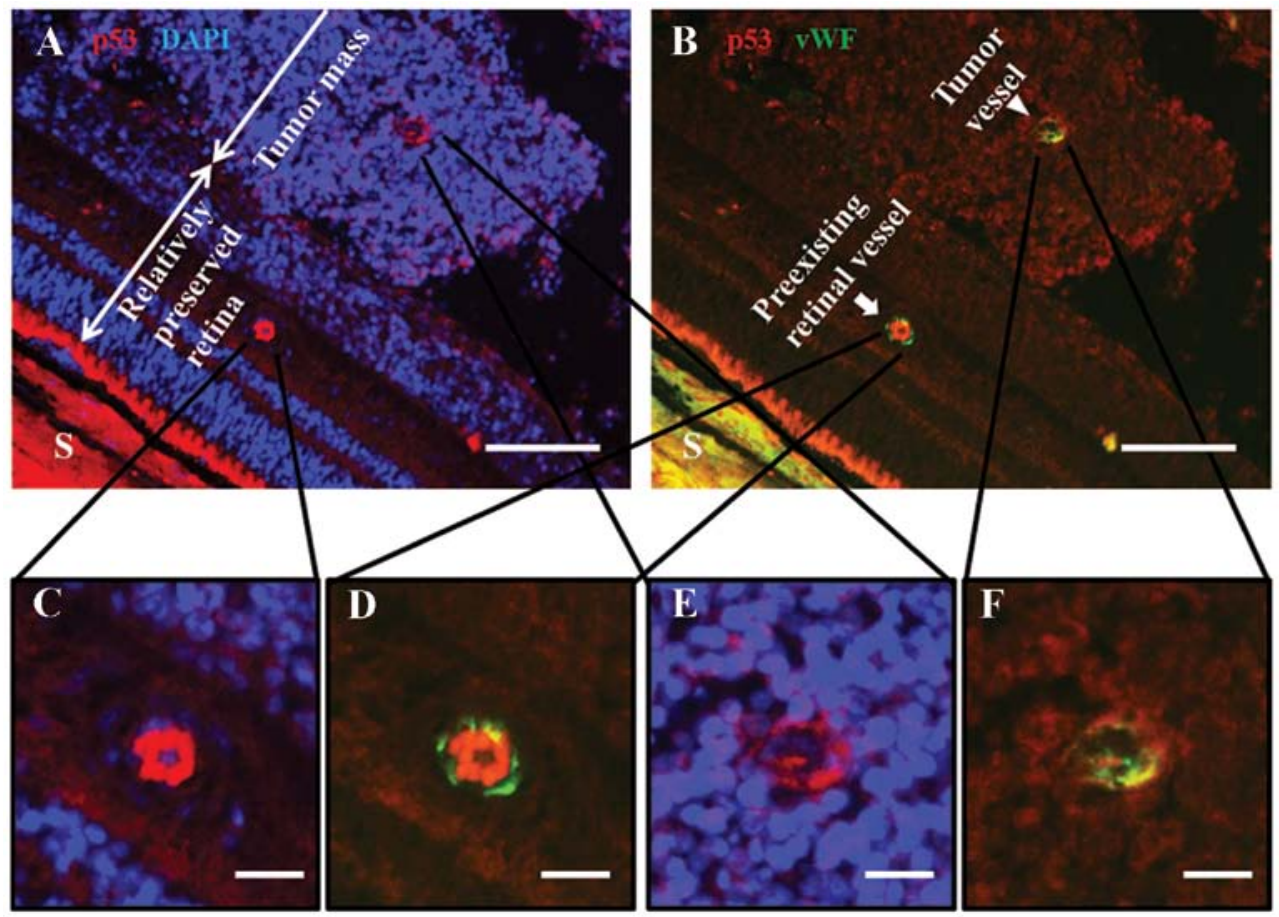

Figure 5. A representative image of a human retinoblastoma specimen which contains both relatively preserved retina and a tumor mass (A and B). When double stained with anti-p53 (red) and anti-vWF (green) antibodies, vWF-positive vascular endothelium of presumedly preexisting retinal vessels found in relatively preserved retina did not exhibit any p53 immunopositivity (C and D). In contrast, vWF-positive vascular endothelium in the tumor mass demonstrated simultaneous p53 immunoreactivity (E and F). S, sclera; vWF, von Willebrand factor. Scale bars, A and B, $100 \mu \mathrm{m}$; C-F, $20 \mu \mathrm{m}$.

Cultured retinoblastoma cell lines are known to show cytoplasmic or mixed nuclear and cytoplasmic distribution of the p53 protein in vitro. When stained with the p1801 monoclonal antibody, the p53 protein exhibits a cytoplasmic localization in Y79 cells (10). However, in the present study, both Y79 and SNUOT-Rb1 cell induced orthotopic retinoblastoma models 
consistently demonstrated nuclear accumulation of p53 in the tumor cells. It has been reported that the gene expression pattern of xenografted human glioma cells is different from that of cells grown in vitro (21). A previous study which compared the gene expression profile of human pancreatic cancer cells cultured in ectopic and orthotopic conditions revealed that the organ microenvironment is an important determinant of the gene expression profile (22). The authors postulated that the tumor microenvironment of the orthotopic retinoblastoma model may have induced the molecular change which affects the nuclear localization signal of the p53 protein.

Tumor-associated endothelial cells which share the same genetic aberration of the original tumor have been noted in several tumor types such as lymphoma and renal carcinoma $(23,24)$. More recently, direct evidence for the presence of tumor cell-derived tumor-associated microvasculature has been noted in human malignancies such as glioblastoma and neuroblastoma (6-8). To the best of our knowledge, this is the first report concerning a cytogenetic abnormality of the tumor vascular endothelium in human and orthotopic models of retinoblastoma. Moreover, endothelial immunoreactivity against anti-p53 antisera found in the orthotopic retinoblastoma model could be a strong evidence for the direct contribution of retinoblastoma cells to tumor vascularization.

The ratio of tumor-derived microvessels has been reported to be as high as $78 \%$ in human neuroblastoma (6). According to a report which analyzed tumor-specific chromosomal aberration by FISH assay in tumor vascular endothelium of glioblastoma, $60.7 \%$ (ranging from 20 to 90\%) of tumor endothelial cells showed chromosomal aberration (7). In the present study, the ratio of p53 nuclear accumulation among mature tumor vascular endothelium was $32.9 \%$ (ranging from 31.3 to $33.8 \%$ ). Although this is a pilot study involving only a small number of cases, our results showed a relatively low mean value and small inter-subject variability compared to that of previous studies on glioblastoma. In our opinion, the lower mean value in this study could have been influenced by a low sensitivity of p53 immunostaining for the detection of cytogenetic abnormalities. The small inter-subject variability may be related to the homogeneous clinicopathological features of our participants.

Briefly, p53 nuclear accumulation in some of the mature tumor endothelium as well as in the tumor cells was consistently observed in both the human and orthotopic models of retinoblastoma. Our results imply a role of retinoblastoma cells in tumor angiogenesis and this feature should be considered in future vascular targeted therapy of retinoblastoma. A discrepancy in the ratio of p53 immunopositivity between the SNUOT-Rb1 and Y79 cell induced orthotopic model observed in this study may reflect the different biological properties of each cell line. Previously our group demonstrated that the doubling time of SNUOT-Rb1 cells was shorter than Y79 cells (13), and the tumor growth pattern was relatively more aggressive in the SNUOT-Rb1 cell model. During tumor vascularization, the tumor vascular endothelium is known to originate from neighboring capillary (25) and bone marrowderived precursor cells (26). More recent studies revealed the role of endothelial differentiation of tumor stem-like cells in tumor angiogenesis. A more rapidly growing tumor needs an abundant vascular supply, and if we assume that the endothe- lial differentiation capacity of tumor stem-like cells is limited to a certain degree, the proportion of tumor endothelium which is derived from tumor stem-like cells could be lower in rapidly growing tumors. Further study comparing the microvascular density in both orthotopic retinoblastoma models could provide a better understanding in regards to the role of p53-positive endothelium in tumor vascularization.

\section{Acknowledgements}

This study was supported by the Bio-Signal Analysis Technology Innovation Program (2009-0090895), the Pioneer Research Program (2012-0009544), the Global Core Research Center (GCRC) grant from NRF/MEST, Korea (20120001187), the Seoul National University Research Grant (800-20130338), and the Seoul National University Hospital Research Grant (04-2013-0520). We thank Ms. Esther Yang for the technical assistance.

\section{References}

1. Burnier MN, McLean IW, Zimmerman LE and Rosenberg SH: Retinoblastoma. The relationship of proliferating cells to blood vessels. Invest Ophthalmol Vis Sci 31: 2037-2040, 1990.

2. Rössler J, Dietrich T, Pavlakovic H, et al: Higher vessel densities in retinoblastoma with local invasive growth and metastasis. Am J Pathol 164: 391-394, 2004.

3. Jockovich ME, Murray TG, Escalona-Benz E, Hernandez E and Feuer W: Anecortave acetate as single and adjuvant therapy in the treatment of retinal tumors of $\mathrm{LH}_{\mathrm{BETA}} \mathrm{T}_{\mathrm{AG}}$ mice. Invest Ophthalmol Vis Sci 47: 1264-1268, 2006.

4. Lee SY, Kim DK, Cho JH, Koh JY and Yoon YH: Inhibitory effect of bevacizumab on the angiogenesis and growth of retinoblastoma. Arch Ophthalmol 126: 953-958, 2008.

5. Yang H, Cheng R, Liu G, et al: PEDF inhibits growth of retinoblastoma by anti-angiogenic activity. Cancer Sci 100: 2419-2425, 2009.

6. Pezzolo A, Parodi F, Corrias MV, Cinti R, Gambini C and Pistoia V: Tumor origin of endothelial cells in human neuroblastoma. J Clin Oncol 25: 376-383, 2007.

7. Ricci-Vitiani L, Pallini R, Biffoni M, et al: Tumour vascularization via endothelial differentiation of glioblastoma stem-like cells. Nature 468: 824-828, 2010.

8. Wang R, Chadalavada K, Wilshire J, et al: Glioblastoma stem-like cells give rise to tumour endothelium. Nature 468: 829-833, 2010.

9. Nork TM, Poulsen GL, Millecchia LL, Jantz RG and Nickells RW: p53 regulates apoptosis in human retinoblastoma. Arch Ophthalmol 115: 213-219, 1997.

10. Schlamp CL, Poulsen GL, Nork TM and Nickells RW: Nuclear exclusion of wild-type p53 in immortalized human retinoblastoma cells. J Natl Cancer Inst 89: 1530-1536, 1997.

11. Seigel GM, Hackam AS, Ganguly A, Mandell LM and Gonzalez-Fernandez F: Human embryonic and neuronal stem cell markers in retinoblastoma. Mol Vis 13: 823-832, 2007.

12. Zhong X, Li Y, Peng F, et al: Identification of tumorigenic retinal stem-like cells in human solid retinoblastomas. Int J Cancer 121: 2125-2131, 2007.

13. Kim JH, Kim JH, Yu YS, Kim DH, Kim CJ and Kim KW: Establishment and characterization of a novel, spontaneously immortalized retinoblastoma cell line with adherent growth. Int J Oncol 31: 585-592, 2007.

14. Reid TW, Albert DM, Rabson AS, et al: Characteristics of an established cell line of retinoblastoma. J Natl Cancer Inst 53: 347-360, 1974.

15. Kato MV, Shimizu T, Ishizaki K, et al: Loss of heterozygosity on chromosome 17 and mutation of the $\mathrm{p} 53$ gene in retinoblastoma. Cancer Lett 106: 75-82, 1996.

16. Laurie NA, Donovan SL, Shih CS, et al: Inactivation of the $p 53$ pathway in retinoblastoma. Nature 444: 61-66, 2006.

17. Danovi D, Meulmeester E, Pasini D, et al: Amplification of Mdmx (or $M d m 4$ ) directly contributes to tumor formation by inhibiting p53 tumor suppressor activity. Mol Cell Biol 24: 5835-5843, 2004. 
18. Lu F, Chi SW, Kim DH, Han KH, Kuntz ID and Guy RK: Proteomimetic libraries: design, synthesis, and evaluation of p53-MDM2 interaction inhibitors. J Comb Chem 8: 315-325, 2006.

19. Pokroy R, Tendler Y, Pollack A, Zinder O and Weisinger G: p53 expression in the normal murine eye. Invest Ophthalmol Vis Sci 43: 1736-1741, 2002.

20. Weisinger G, Tendler Y and Zinder O: Quantification of p53 expression in the nervous system. Brain Res Brain Res Protoc 6: 71-79, 2000.

21. Camphausen K, Purow B, Sproull M, et al: Influence of in vivo growth on human glioma cell line gene expression: convergent profiles under orthotopic conditions. Proc Natl Acad Sci USA 102: 8287-8292, 2005.

22. Nakamura T, Fidler IJ and Coombes KR: Gene expression profile of metastatic human pancreatic cancer cells depends on the organ microenvironment. Cancer Res 67: 139-148, 2007.
23. Fonsato V, Buttiglieri S, Deregibus MC, Puntorieri V, Bussolati B and Camussi G: Expression of Pax 2 in human renal tumor-derived endothelial cells sustains apoptosis resistance and angiogenesis. Am J Pathol 168: 706-713, 2006.

24. Streubel B, Chott A, Huber D, et al: Lymphoma-specific genetic aberrations in microvascular endothelial cells in B-cell lymphomas. N Engl J Med 351: 250-259, 2004.

25. Holash J, Maisonpierre PC, Compton D, et al: Vessel cooption, regression, and growth in tumors mediated by angiopoietins and VEGF. Science 284: 1994-1998, 1999.

26. Lyden D, Hattori K, Dias S, et al: Impaired recruitment of bonemarrow-derived endothelial and hematopoietic precursor cells blocks tumor angiogenesis and growth. Nat Med 7: 1194-1201, 2001. 